\title{
Thoracic Actinomycosis - Report of a Patient with Advanced-Stage Disease
}

\author{
José Wellington Alves dos Santos, Ricardo Morgental Zambenedetti, Keli Cristina Mann and Luiz Fenando Ximenes Cibin \\ Pulmonary Disease Service, Santa Maria University Hospital; Santa Maria, RS, Brazil
}

\begin{abstract}
The authors describe a case of advanced thoracic actinomycosis. A 44-year-old man with previous episodes of productive cough with purulent sputum, fever, chest pain and subcutaneous nodules on the left hemithorax was admitted with complaints of lumbar pain irradiating into left lower limb and presence of mass lesion on the left lumbar region. Imaging evaluations were obtained and the patient underwent left lower lobectomy. Histopathology, microscopic examination and anaerobic culture of the resected material diagnosed thoracic actinomycosis. Key-Words: Actinomycosis, thoracic, advanced.
\end{abstract}

Actinomycosis is a rare chronic infection caused by species of Actinomyces, characterized by abscess formation, tissue fibrosis, suppurative lesions and fistulas with purulent discharge containing sulphur granules [1-3]. Due to its multiform manners of presentation and non-specificity from its clinical features, it has been considered a challenging diagnosis [4-7].

This report describes a case of advanced thoracic actinomycosis in a 44-year-old man.

\section{Case Report}

A 44-year-old otherwise healthy man, who had a 15 packyear history of smoking cigarettes, developed productive cough with purulent sputum, fever, and pain on the left hemithorax. He was treated receiving antibiotic therapy achieving partial improvement of symptoms. Two months later, painful subcutaneous nodules appeared on the left posterior hemithorax. One year and nine months later, he presented new picture of fever, productive cough and pain on the left hemithorax, following irregular antibiotic treatment. He underwent drainage of subcutaneous nodules and developed fistulas on the postoperative wound. Seven months later he was first admitted to our hospital for diagnostic investigation due to radiological lesion (consolidation) on the lower lobe of the left lung. Thoracic transcutaneous biopsy performed detected no evidence of neoplasia or infection. The patient denied further procedures to establish a diagnosis and two years later he was admitted to our hospital again presenting complaints of lumbar pain irradiating into left lower limb with associated indurated mass lesion on the left lumbar region (Figure 1). His vital signs were normal. The physical examination disclosed breath sounds suppressed over the lower lobe of the left lung. Laboratory studies revealed a leuckocyte count of 9,000 cells. His serum electrolytes and creatinine levels were both normal. Chest radiograph showed persistence of the previous pulmonary lesion. Computed

Received on 11 September 2006; revised 28 November 2006. Address for correspondence: Dr. José Wellington Alves dos Santos. Rua Venâncio Aires, 2020/ 403, Zip code: 97010-004, Santa Maria, RS, Brazil. Phone number: +55 55 3225-3018. Fax: +55 55 3220-8005. Email: jwasb@terra.com.br.

The Brazilian Journal of Infectious Diseases 2007;11(1):157-159. (C) 2007 by The Brazilian Journal of Infectious Diseases and Contexto Publishing. All rights reserved. tomography (CT) scan of the chest revealed necrosis and consolidation on the left lower lobe with pleural involvement (Figure 2). Magnetic resonance imaging (MRI) scans of the chest and abdomen evidenced a 13 × $9 \mathrm{~cm}$ heterogeneous collection, predominantly liquid, which was found to be attached to the left posterior hemithorax base, diaphragm, retroperitoneum, posterior abdominal wall and paravertebral space, involving thoracic, lumbar, and sacral vertebral bodies. He was submitted to a left lower lobectomy (Figure 3). Histopathology of the resected specimen showed the presence of sulphur granules (Figure 4). Bacterioscopic examination revealed clusters of radiated, filamentous, branching, Grampositive microorganisms stained by Gram-Brown-Brenn (Figure 5). Anaerobic culture of the material identified the growth of Actinomyces israelli. After penicillin treatment for 12 months, the patient has been well and no evidence of recurrent disease is observed.

\section{Discussion}

Actinomycosis is a chronic, suppurative, granulomatous infection caused by anaerobic Gram-positive bacilli from the species Actinomyces, and A. israelli is the most frequent pathogen involving human infection. These microorganisms are usually commensal in the oropharynx, gastrointestinal tract and female genitals, requiring a portal of entry to cause infection. $[1,2,4,8]$. It is a rare disease that attacks men most frequently (3:1), between the fourth and fifth decades of life [4]. Classically, clinical forms involve cervicofacial (55\%), abdominopelvic (20\%), thoracic (15\%) and mixed organs (10\%), including skin, brain, pericardium and extremities $[1,2,4,5,8]$.

Thoracic actinomycosis is generally caused by aspiration of contaminated secretion from the oropharynx or direct extension of cervicofacial or abdominopelvic forms $[1,2,4,7,9]$. The thoracic disease may also occur by lymphohematogenic dissemination. However, it is rare and usually late [1,2,10,11]. The lower lobes are the most commonly involved. Cough, sputum, fever, weight loss, thoracic pain, hemoptysis, and dyspnea are frequent complaints [2,6,9-12].

Contiguous extension from thoracic disease to adjacent structures, or hematogenous spread to the brain, kidneys, genitals, subcutaneous tissues and skin usually occurs in untreated disease evolution. Transdiaphragmatic fistulas or retroperitoneal invasion may attack the abdomen [2,7,11]. 
Figure 1. Multiple scars on the left hemithorax and indurated mass lesion on the left lumbar region.

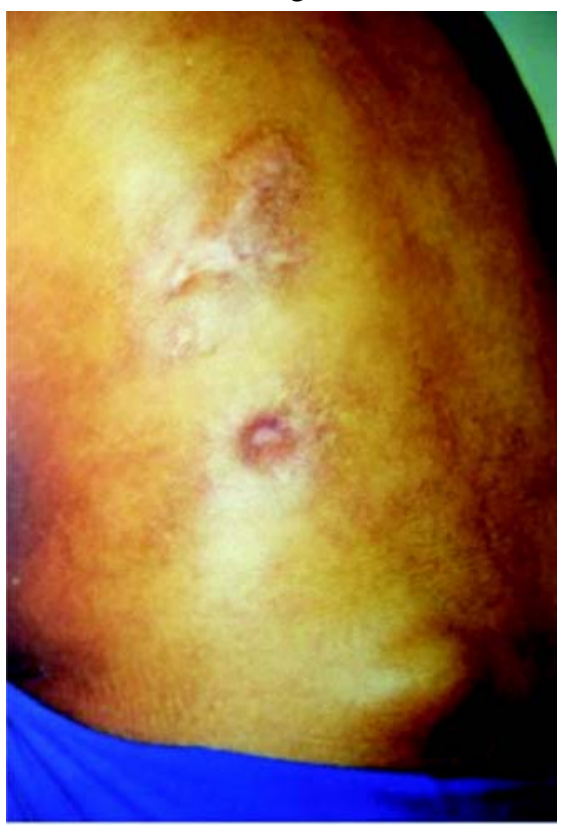

Figure 2. Chest CT: necrosis and consolidation on the left lower lobe with pleural involvement.

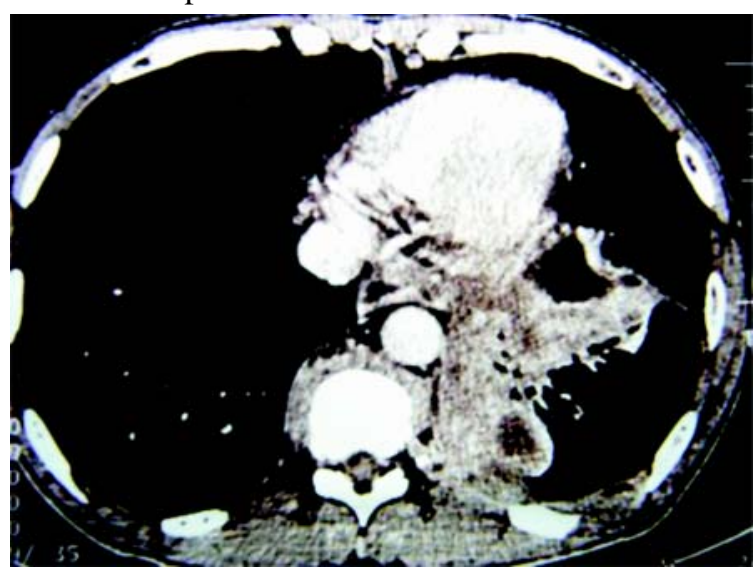

Figure 3. Lobectomy specimen: macroscopic appearance.

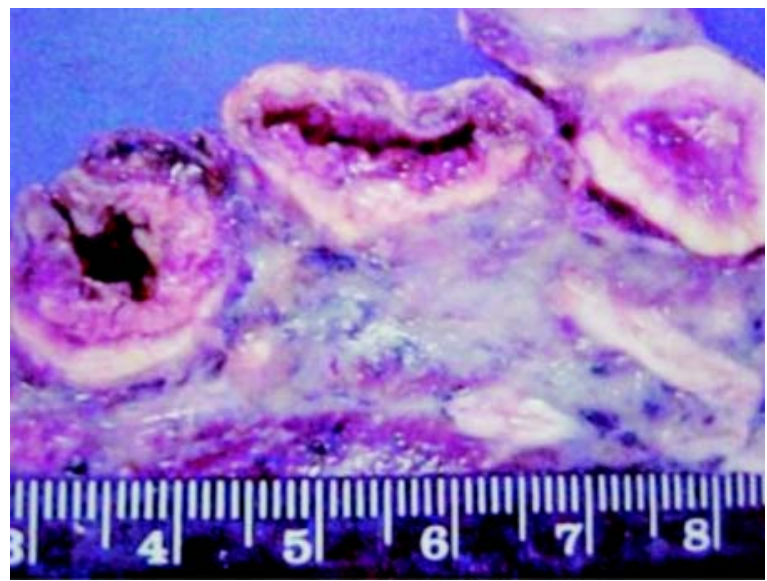

Figure 4. Hematoxylin and Eosin stain: sulphur granule.

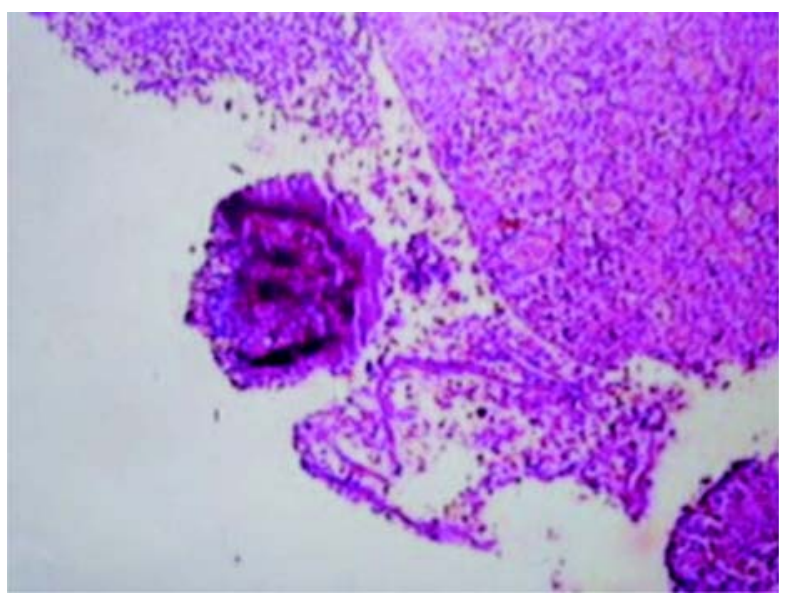

Figure 5. Gram Brown-Brenn stain: clusters of radiated, filamentous, branching, Gram-positive microorganisms.

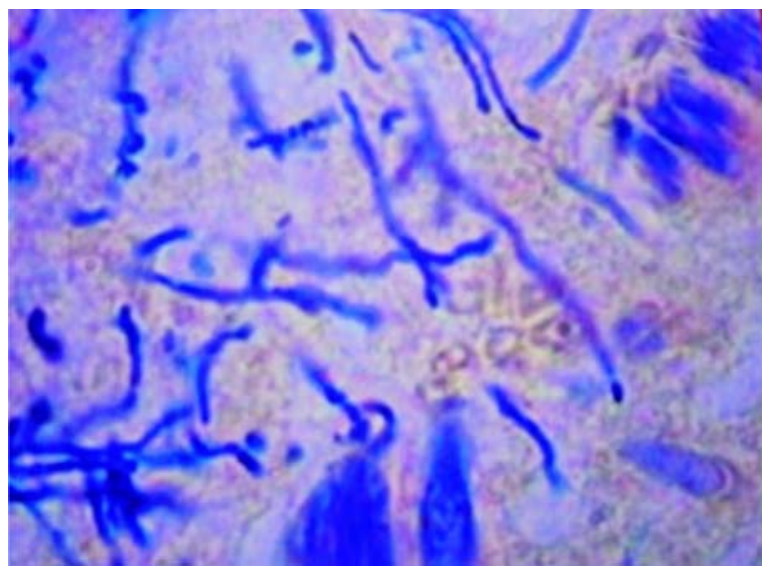

Hepatic or renal abscess and compression of blood vessels or nervous roots may also be observed [13]. Thoracic wall involvement is found in $12 \%$ of cases, leading to bone destruction and osteomyelitis with associated subcutaneous nodules and fistulas draining purulent material [14]. Vertebral involvement is extremely rare and associated spinal cord compression is unusual [15].

The radiographic presentation of thoracic actinomycosis is variable and nonspecific, including pulmonary consolidations, cavitary lesions, and pleural effusion. CT may be useful in demonstrating thoracic wall involvement, bone destruction, presence of osteomyelitis and pleural alterations. Invasion to mediastinum, retroperitoneum and compromising of soft tissues usually associated with fistulas and fibrosis are also radiological findings suggestive of thoracic actinomycosis that may be demonstrated by MRI [13,16-19].

The low clinical suspicion due to insidious clinical presentation makes the diagnosis of thoracic actinomycosis difficult, which is based on bacterioscopic examination or culture of the material aspirated from lesions, or 
histopathological examination of the resected specimens and from bronchoscopic biopsy. Sulphur granules composed by clusters of radiated, filamentous, Grampositive microorganisms are characteristic in compromised tissues. Although the growth of Actinomyces on anaerobic cultures occurs in less than $50 \%$ of cases, it is considered the gold standard method for definitive diagnosis [2,4,69,11,12].

A. israelli is sensible to multiple antibiotics. The treatment of choice is parenteral penicillin $\mathrm{G}$ in high doses (20 million IU/day) for 4 weeks, supplemented by oral penicillin V for 6-12 months. However, in complicated disease, combined clinical treatment and surgical resection of necrotic tissues is the most effective, determining favorable evolution and low recurrence rate $[4,7,11,12]$.

As a conclusion, thoracic actinomycosis should be considered in the differential diagnosis of patients presenting respiratory symptoms of chronic evolution and pulmonary consolidations with associated pleural involvement, extending to the thoracic wall, vertebral bodies or diaphragm. Considering the successful use of antibiotic therapy in preventing surgical procedures and morbidity resulting from advanced-stage disease, the early recognition and clinical treatment is essential for optimal management.

\section{References}

1. Bates M., Cruickshank G. Thoracic actinomycosis. Thorax 1957;12(2):99-124.

2. Smego R.A., Foglia G. Actinomycosis. Clin Infect Dis 1998;26(6):1255-61.

3. Russo T.A. Agents of actinomycosis. In: Mandell G.L. Ed. Principles and Practice of Infectious Disease. 5th edn. New York: Churchill Livingstone, 1995. p. 2645-54.
4. Bennhoff D.F. Actinomycosis: diagnostic and therapeutic considerations and a review of 32 cases. Laryngoscope 1984;94(9):1198-217.

5. Weese W.C., Smith I.M. A study of 57 cases of actinomycosis over a 36-year period. A diagnostic 'failure' with good prognosis after treatment. Arch Intern Med 1975;135(12):1562-8.

6. Tastepe A.I., Ulasan N.G., Liman S.T., et al. Thoracic actinomycosis. Eur J Cardiothorac Surg 1998;14(6):578-83.

7. Mabeza G.F., Macfarlane J. Pulmonary actinomycosis. Eur Respir J 2003;21(3):545- 51.

8. Brown J.R. Human actinomycosis: a study of 181 subjects. Hum Pathol 1973; 4(3):319-30.

9. Hsieh M.J., Liu H.P., Chang J.P., Chang C.H. Thoracic actinomycosis. Chest 1993;104(2):366-70.

10. Apotheloz C., Regamey C. Disseminated infection due to Actinomyces myeri - case report and reveiew. Clin Infect Dis 1996;22(4):621-5.

11. Varkey B., Landis F.B., Tang T.T., Rose H.D. Thoracic Actinomycosis. Dissemination to skin, subcutaneous tissue, and muscle. Arch Intern Med 1974;134(4):689-93.

12. Dujneungkunakorn T., Riantawan P., Tungsagunwattana S. Pulmonary actinomycosis: a study of 16 cases from Central Chest Hospital. J Med Assoc Thai 1999;82(6):531-5.

13. de la Espina M.A.Z., Lopez-Mendez C., Ruiz-Martinez R., MolinoTrinidad C. Pulmonary actinomycosis with thoracic soft tissue mass: a rare onset form. Eur J Radiol 2001;37(3):195-9.

14. Burden P. Actinomycosis. J Infect 1989;19(2): 95-9.

15. Voisin L., Vittecoq, O., Mejjad, O., et al. Spinal abscess and spondylitis due to actinomycosis. Spine 1998;23(4):487-90.

16. Cheon J.E., Im J.G., Kim M.Y., Lee J.S., Choi G.M., Yeon K.M. Thoracic actinomycosis: CT findings. Radiology 1998;209(1):229-33.

17. Wand A., Gilbert H.M., Litvack B., Markisz J.A. MRI of thoracic actinomycosis. J Comput Assist Tomogr 1996;20(5):770-2.

18. Allen H.A., Scatarige J.C., Kim M.H. Actinomycosis: CT findings in six patients. Am J Roentgenol 1987;149(6):1255-8.

19. Yung B.C., Cheng J.C.K., Chan T.F., et al. Aggressive thoracic actinomycosis complicated by vertebral osteomyelitis and epidural abscess leading to spinal cord compression. Spine 2000;25(6):745-8. 